\title{
Mitochondrial factor and cell cytokines associate with TCM syndrome scale in vascular dementia patients
}

\author{
LINJUAN SUN \\ Department of Neurology, Xiyuan Hospital, China Academy of Chinese Medical Sciences, \\ Haidian, Beijing 100091, P.R. China
}

Received June 23, 2017; Accepted October 16, 2017

DOI: $10.3892 /$ etm.2017.5387

\begin{abstract}
Mitochondrial factor and cell cytokines play important roles in the incidence of vascular dementia (VD), but their correlations with inflammatory and mitochondrial factors and the role of both in the kidney essence deficiency pattern and phlegm turbidity blocking orifice pattern are not clear. This study was aimed at studying the correlations between the serum mitochondrial factor and cell cytokines with TCM Syndrome Scale in vascular dementia. According to the inclusion criteria we collected 108 vascular dementia patients which were divided into the kidney essence deficiency pattern and phlegm turbidity blocking orifice pattern based on the TCM Syndrome Scale. We measured serum tumor necrosis factor- $\alpha$ (TNF- $\alpha$ ), interleukin-6 (IL-6), interleukin-18 (IL-18) concentration using an enzyme-linked immunosorbent assay (ELISA) and serum malondialdelyde (MDA) and superoxide dismutase (SOD) was quantified according to instructions of kits from the 108 patients (45 in kidney essence deficiency pattern, 63 in phlegm turbidity blocking orifice pattern). The scale scores were assessed using TCM Syndrome Scale, MMSE, Hachinski, Barthel, BBS, CDR. There was a significant difference on the scores of CDR and the factors of the memory, judgment, social affairs, personal care, family and hobbies among the two groups, The means of kidney essence deficiency group was higher than that of phlegm turbidity blocking orifice pattern group. IL-18 and SOD in the phlegm turbidity blocking orifice group was higher than those in the kidney essence deficiency pattern; IL-6 in phlegm turbidity blocking orifice pattern group was lower than that in the kidney essence deficiency pattern. By logistic regression analysis, we demonstrated that high concentration of IL- 6 , TNF- $\alpha$, and MDA were associated with increased TCM syndrome scores in two groups, while IL-6, IL-18, TNF- $\alpha$, SOD were associated
\end{abstract}

Correspondence to: Dr Linjuan Sun, Department of Neurology, Xiyuan Hospital, China Academy of Chinese Medical Sciences, 1 Xiyuan Caochang, Haidian, Beijing 100091, P.R. China

E-mail:sip8h5@163.com

Keywords: vascular dementia, TCM Syndrome Scale, mitochondrial factor, cell cytokines with decreased MMSE, Barthel. Our study support the notion that IL-6 plays a more important role in the integral of kidney essence deficiency pattern, IL-18 is a more important factor in phlegm turbidity blocking orifice pattern. The results can be used as the theoretical basis of traditional Chinese Medicine prescription and pharmacological research in future, through decrease in IL-6 lever and increase in SOD in integral kidney essence deficiency pattern, and IL-18 as the main anti inflammation index in phlegm turbidity blocking orifice pattern.

\section{Introduction}

Vascular dementia (VD) is clinical syndrome that encompasses a wide spectrum of cognitive disorders caused by cerebrovascular disease. VD patients perform a series of abnormities such as slowed thinking, forgetfulness, depression and anxiety, and disorientation. The patients manifest defective executive functions such as problem solving, working memory, thinking, reasoning, judgment and planning. VD is considered to be the second most common cause of cognitive impairment after Alzheimer's disease in the elderly (1). Although it is important to understand the pathophysiology of the disease, the underlying reason of pathophysiology of the disease remains unknown. More and more evidence shows that inflammatory reaction and mitochondrial damage are two important mechanisms of VD after stroke. Oxidation is induced by hypoxia leading to mitochondrial dysfunction, neuronal damage and apoptosis, malondialdehyde content, release of reactive oxygen species and free radicals (2-5). Vascular inflammatory factors trigger endothelial dysfunction, and increases neuroinflammatory response (6). Inflammatory factors such as interleukins: interleukin-6 (IL-6); interleukin-1 (IL-1); interleukin-18 (IL-18), TNF- $\alpha$, and C-reactive protein (CPR) (7).

The kidney essence deficiency pattern and phlegm turbidity blocking orifice pattern obtained by the study on TCM syndromes distribution regularity are representative asthenia syndrome and sthenia syndrome in VD. Kidney is the source of acquired constitution, governing bones and producing marrow. Kidney $q i$ contact with the brain that is fu-viscera of mental activity, which controls the human higher nervous activities. At septuple eight, with kidney $q i$ asthenic, Yang Ming pulse week, and Tian Gui exhausted, brain marrow is deprived of nourishment, which result in the 
decline of behavior and ability of daily living. In addition, the phlegm is produced by qi stagnation, spleen failing to transport and transform which produced by the stagnation of liver qi, kidney deficiency, yin and yang deficiency of kidney, lung $q i$ disorder. Phlegm is yin evil that disturb the fu-viscera of mental activity, repress the Qing Yang rising, and it could be mixed with other tangible pathogenic products, producing new etiology and pathogenesis such as the fusion of phlegm stasis in brain, the mental confusion caused by wind phlegm, the damaged brain results of phlegm fire, collectively leading to brain malnutrition.

Mitochondrial factor and cell cytokines play important role in the incidence of VD, but their correlations with inflammatory factors and mitochondrial factors and the role of both in the kidney essence deficiency and phlegm turbidity blocking orifice pattern are not clear. This study was aimed at studying the correlations between the serum mitochondrial factor and cell cytokines with TCM Syndrome Scale in vascular dementia.

\section{Materials and methods}

Experimental procedures. This study was a comparative trial with two arms to analyse the relevance of TCM Syndrome Scale with Mitochondrial factor and cell cytokines in VD. The trial was performed at Xiyuan hospital of China Academy of Chinese Medical Sciences. All patients evaluated at our neurological clinic from January 2015 to December 2016 for suspected MMSE scores were considered eligible for the study. The participants were divided into two groups that including phlegm turbidity blocking orifice pattern and kidney essence insufficiency pattern of VD according to the TCM symptoms scale. Vascular dementia symptom score (SDSVD), named TCM symptoms scale, has a total score of 30 points. If the score was between 7 and 14 points, it would correspond to mild dementia, scores $15-22$ points were moderate dementia, scores 23-30 points were severe dementia. Cognitive functions were assessed using the China-MMSE (Mini-mental state examination). The ability of daily life were assessed using the ADL (activities of daily living). Behavior ability used the BBS (Blessed Behavior Scale); Clinical Dementia Rating (CDR) was also used to assess the Cognitive changes. This trial was carried out according to the standards of the International Committee on Harmonization on GCP and revised version of the Declaration of Helsinki. The study protocol was approved by the Ethics Committees of Xiyuan Hospital of China Academy of Chinese Medical Sciences, and Institutional Review Boards at Xiyuan Hospital of China Academy of Chinese Medical Sciences.

Sample size calculation. The sample size was based on formula as follows: $\mathrm{n}=2 \times 2+0.25 \mu \alpha 2$ by bilateral $\alpha=0.05$, $1-\beta=0.8, \delta / \sigma=0.6$, according to the calculation formula, total of 90 participants (45 participants per arm) will be needed.

Diagnostic criteria for vascular dementia. The diagnostic criteria of Western medicine: diagnostic criteria for vascular dementia that was according to Diagnostic and Statistical Manual of Mental Disorders (DSM-IV), published in the 1994 by American Psychiatric Association. The diagnostic criteria of traditional Chinese medicine: the standard of syndrome differentiation of vascular dementia (SDSVD): the total score was 30 points, if the score was more than 7 points, it could be involved in the syndrome. Inclusion criteria are shown below: a) met the diagnostic criteria of the clinical standard of vascular dementia; b) the dementia occured within 3 months after the stroke, and lasting for 3 months; c) accord to the TCM syndrome differentiation scale of vascular dementia (SDSVD), the integral of the syndrome was more than 7 points; d) the score of Folstein MMSE was less than 26 points; e) the scoring criteria of the HIS was more than 7 points; f) exclude dementia caused by other reasons; g) agreed with the study protocol and signed informed consent. Exclusion criteria are shown below: Participants who met any of the following were excluded: a) Alzheimer's disease (AD) or other types of dementia patients; b) difficulties in attending the trial (e.g. severe heart, lung, renal dysfunction, severe diabetes mellitus and the history of mental disease); c) patients who did not meet the inclusion criteria, unable to distinguish the type of syndrome, and data incomplete; d) refused to sign the informed consent.

\section{Multiplex arrays}

Blood samples collection. Blood samples $(5 \mathrm{ml})$ were collected from the antecubital vein of each participant after an overnight fast. The samples were allowed to clot for $1 \mathrm{~h}$ at room temperature. After centrifugation at $3000 \mathrm{rpm}$ for $15 \mathrm{~min}$, serum was extracted from the collected blood samples, placed into micro tubes and stored at $-80^{\circ} \mathrm{C}$ until analysis.

Measurement of oxidative stress parameters. SOD activity was detected based on its ability to inhibit the superoxide anion free radical of $\mathrm{O}_{2}$-generated by the xanthine/xanthine oxidase system. The absorbance at $550 \mathrm{~nm}$ was monitored and SOD levels were expressed as $\mathrm{U} / \mathrm{ml}$.

Quantification of MDA level. The concentration of serum malondialdelyde (MDA) was quantified according to instructions using thiobarbituric acid (TBA) reagent, and the absorbance of the supernatant was measured spectrophotometrically at $530 \mathrm{~nm}$. The concentration of MDA was expressed as $\mathrm{nmol} / \mathrm{ml}$.

Assay of serum TNF- $\alpha, I L-6, I L-18$. We detected the serum levels of TNF- $\alpha$, IL- 6 and IL-18 using commercial enzyme linked immunosorbent (ELISA) kits (eBioscience, San Diego, CA, USA), while no cross-reactivity was detected. Standard curve concentration were calculated in duplicate for each plate. All samples were assayed in duplicate. Absorbency was measured using an iMark microtiter plate reader (Bio-Rad Laboratories, Inc., Hercules, CA, USA) set at $450 \mathrm{~nm}$. The expression of TNF- $\alpha$, IL- 6 , IL-18 concentration was recorded as $\mathrm{pg} / \mathrm{ml}$.

Statistical analysis. Demographic variables were assessed using t-tests for continuous variables and Chi-square tests for categorical variables. Continuous variables were expressed as mean (SD) or median (range) when necessary. Means of TCM Syndrome Scale, MMSE, ADL, BBS, CDR mitochondrial factor and cell cytokines were compared by independent t-test. Pearson correlation analysis was used to assess the correlation between TCM Syndrome Scale, MMSE, ADL, BBS, 
Table I. General characteristics of the enrollment demography.

\begin{tabular}{lcc}
\hline Parameters & $\begin{array}{c}\text { Kidney essence } \\
\text { deficiency } \\
\text { group }(\mathrm{n}=45)\end{array}$ & $\begin{array}{c}\text { Phlegm turbidity } \\
\text { blocking orifice } \\
\text { pattern group (n=63) }\end{array}$ \\
\hline Age group 1 $(<59)$ & 3 & 8 \\
Age group 2 $(60-69)$ & 10 & 20 \\
Age group 3 $(70-79)$ & 15 & 25 \\
Age group 4 $(>80)$ & 17 & 10 \\
Male & 27 & 31 \\
Female & 18 & 32 \\
\hline
\end{tabular}

Table II. The Comparison of means of six scale scores in two groups.

\begin{tabular}{lccc}
\hline $\begin{array}{l}\text { Six scale } \\
\text { scores }\end{array}$ & $\begin{array}{c}\text { Kidney essence } \\
\text { deficiency group }\end{array}$ & $\begin{array}{c}\text { Phlegm turbidity } \\
\text { blocking } \\
\text { orifice pattern }\end{array}$ & P-value \\
\hline MMSE & $14.20 \pm 6.185$ & $16.16 \pm 4.209$ & 0.053 \\
TCM symptom & $23.56 \pm 7.458$ & $23.87 \pm 6.346$ & 0.812 \\
score & $13.3 \pm 5.3294$ & $11.637 \pm 6.3988$ & 0.158 \\
BBS & $1.4 \pm 0.8568$ & $0.96 \pm 0.5485$ & $0.002^{\text {a }}$ \\
CDR & $13.44 \pm 1.949$ & $13.95 \pm 1.782$ & 0.163 \\
Hachinski & $61.44 \pm 26.277$ & $65.25 \pm 26.391$ & 0.460 \\
Barthel & & & \\
\hline
\end{tabular}

${ }^{\mathrm{a}} \mathrm{P}<0.01$.

CDR with mitochondrial factor and cell cytokines in each group. In order to test the most important influencing factor in mitochondrial factor and cell cytokines on TCM Syndrome Scale by linear regression analysis was employed. $\mathrm{P}<0.05$ was considered to indicate a statistically significant difference. Data were analyzed by the Statistical Package for the Social Sciences software (SPSS 16.0 for Windows).

\section{Results}

There was no significant difference on the enrollment demographics between the groups. This study included 108 cases, the group of the kidney essence insufficiency pattern included 45 cases; the phlegm turbidity blocking orifice pattern group had 63 cases. No loss or exclusion. Kidney essence deficiency group included 27 males, 18 females, the maximum age was 85 years, the minimum age was 49 years; the number of patients whose age was less than 59 years was 3 cases; There were 10 cases whose age was 60 to 69 years; there were 15 cases whose age was 70-79 years; the age of 17 cases in the group were more than 80 years. The group of phlegm turbidity blocking orifice pattern included 31 male cases, 32 female cases, the maximum age was 86 years, the minimum age was 50 years. The number of patients whose age was less than 59 years were 8 cases; there were 20 cases whose age was 60 to 69 years; there were 25 cases whose age was
Table III. The Comparison of means of factors of CDR in two groups.

\begin{tabular}{lrcc}
\hline $\begin{array}{l}\text { Factors } \\
\text { of CDR }\end{array}$ & $\begin{array}{c}\text { Kidney essence } \\
\text { deficiency group }\end{array}$ & $\begin{array}{c}\text { Phlegm turbidity } \\
\text { blocking } \\
\text { orifice pattern }\end{array}$ & P-value \\
\hline Memory & $1.278 \pm 0.7116$ & $0.921 \pm 0.5404$ & $0.004^{\mathrm{a}}$ \\
$\begin{array}{l}\text { Discretion } \\
\text { Social affairs }\end{array}$ & $1.3 \pm 0.8216$ & $0.929 \pm 0.5525$ & $0.006^{\mathrm{a}}$ \\
Family+Hobby & $1.644 \pm 0.9512$ & $1.127 \pm 0.798$ & $0.003^{\mathrm{a}}$ \\
Personal care & $1.611 \pm 0.8975$ & $1.198 \pm 0.6447$ & $0.006^{\mathrm{a}}$ \\
\hline
\end{tabular}

${ }^{\mathrm{a}} \mathrm{P}<0.01$.

Table IV. The comparison means of IL-6, IL-18, SOD, MDA, TNF- $\alpha$ in the groups.

\begin{tabular}{lccc}
\hline $\begin{array}{l}\text { Mitochondrial } \\
\text { factor and cell } \\
\text { cytokines }\end{array}$ & $\begin{array}{c}\text { Kidney essence } \\
\text { deficiency } \\
\text { pattern group }\end{array}$ & $\begin{array}{c}\text { Phlegm turbidity } \\
\text { blocking } \\
\text { orifice pattern }\end{array}$ & P-value \\
\hline IL-6 & $13.149 \pm 11.148$ & $8.007 \pm 4.206$ & $0.001^{\text {b }}$ \\
IL-18 & $25.552 \pm 10.783$ & $38.586 \pm 25.074$ & $0.001^{\text {b }}$ \\
TNF- $\alpha$ & $10.491 \pm 9.052$ & $10.227 \pm 7.459$ & 0.868 \\
SOD & $98.390 \pm 17.837$ & $107.09 \pm 20.736$ & $0.025^{\text {a }}$ \\
MDA & $3.439 \pm 0.923$ & $3.511 \pm 1.400$ & 0.764 \\
\hline
\end{tabular}

${ }^{\mathrm{a}} \mathrm{P}<0.05,{ }^{\mathrm{b}} \mathrm{P}<0.01$.

70-79 years; the age of 10 cases in the group were more than 80 years. There was no difference in the two groups on sex ratio $(\mathrm{P}=0.181)$. The ratio of age group in two groups had no difference $(\mathrm{P}=0.068)$. There was no significant difference on the enrollment demographics between the groups, so the two groups have comparability (Table I).

The comparison of means between TCM Syndrome Scale, MMSE, Hachinski, Barthel, BBS, CDR in two groups. Table II carry Student's test in two groups, and compare the mean on each scale. The results showed there was no difference for the total means on MMSE, BBS, Barthel Index, TCM syndrome and HIS. There was a significant difference on the scores of CDR and the factors of the memory, judgment, social affairs, personal care, family and hobbies among the two groups. The means of kidney essence deficiency group was higher than that of phlegm turbidity blocking orifice pattern group (Table III).

IL-6 was higher in kidney essence insufficiency pattern, but IL-18, and SOD were higher in phlegm turbidity blocking orifice pattern group. The plasma levels of the mitochondrial factor and cell cytokines in the two groups are reported in Table IV. The means of IL-18 (38.586 \pm 25.074$)$ and SOD $(107.09 \pm 20.736)$ in the phlegm turbidity blocking orifice pattern group was higher than IL-18 (25.552 \pm 10.783$)$ and SOD 
Table V. The correlation between the mitochondrial factor, cell cytokines and the six scale scores in two groups.

\begin{tabular}{|c|c|c|c|c|c|c|}
\hline Group & & IL-6 & IL-18 & TNF- $\alpha$ & SOD & MDA \\
\hline \multirow{6}{*}{$\begin{array}{l}\text { Kidney essence deficiency } \\
\text { group }\end{array}$} & MMSE & $-0.590^{b}$ & -0.186 & $-0.544^{\mathrm{b}}$ & 0.231 & -0.214 \\
\hline & TCM symptom score & $0.638^{\mathrm{b}}$ & 0.034 & $0.634^{\mathrm{b}}$ & $-0.408^{b}$ & $0.315^{\mathrm{a}}$ \\
\hline & $\mathrm{BBS}$ & $0.293^{\mathrm{a}}$ & $0.567^{b}$ & 0.219 & -0.244 & $0.333^{\mathrm{a}}$ \\
\hline & Barthel & $-0.510^{\mathrm{b}}$ & $-0.331^{\mathrm{a}}$ & $-0.349^{a}$ & 0.146 & -0.272 \\
\hline & CDR & $0.479^{b}$ & $0.446^{\mathrm{b}}$ & 0.283 & -0.285 & $0.407^{\mathrm{b}}$ \\
\hline & Hachinski & 0.114 & $-0.416^{\mathrm{b}}$ & 0.192 & 0.023 & -0.05 \\
\hline \multirow{6}{*}{$\begin{array}{l}\text { Phlegm turbidity blocking } \\
\text { orifice group }\end{array}$} & MMSE & -0.075 & -0.042 & -0.203 & $0.306^{\mathrm{a}}$ & -0.186 \\
\hline & TCM symptom score & $0.270^{\mathrm{a}}$ & $-0.604^{b}$ & $0.556^{\mathrm{b}}$ & $-0.59^{b}$ & $0.324^{\mathrm{b}}$ \\
\hline & BBS & 0.117 & $0.723^{b}$ & -0.186 & $0.311^{\mathrm{a}}$ & -0.071 \\
\hline & Barthel & -0.186 & $-0.569^{b}$ & $-0.070^{\mathrm{a}}$ & -0.100 & -0.117 \\
\hline & CDR & -0.71 & 0.204 & 0.071 & -0.003 & -0.014 \\
\hline & Hachinski & 0.042 & $-0.70^{\mathrm{b}}$ & $0.338^{\mathrm{b}}$ & $-0.406^{\mathrm{b}}$ & 0.159 \\
\hline
\end{tabular}

${ }^{\mathrm{a}} \mathrm{P}<0.05,{ }^{\mathrm{b}} \mathrm{P}<0.01$.

Table VI. Multiple linear regression analysis on TCM syndrome score in the kidney essence deficiency group.

\begin{tabular}{lrrrrr}
\hline \multicolumn{7}{c}{ Model } & \multicolumn{2}{c}{$\begin{array}{c}\text { Non-standard } \\
\text { coefficient }\end{array}$} & & & \\
\cline { 2 - 3 } & B & $\begin{array}{c}\text { Standard } \\
\text { error }\end{array}$ & $\begin{array}{c}\text { Standard } \\
\text { coefficient }\end{array}$ & t-value & P-value \\
\hline Quantity & 33.085 & 5.441 & & 6.080 & $<0.05$ \\
IL-6 & 0.309 & 0.083 & 0.461 & 3.708 & 0.001 \\
SOD & -0.138 & 0.052 & -0.330 & -2.654 & 0.011 \\
\hline
\end{tabular}

Dependent variable: TCM syndrome score in kidney deficiency group.

$(98.390 \pm 17.837)$ in the kidney essence insufficiency pattern. The means of IL- 6 had obvious difference in two groups. The means of IL-6 $(8.007 \pm 4.206)$ in phlegm turbidity blocking orifice pattern group was lower than IL-6 (13.149 \pm 11.148$)$ of the kidney essence insufficiency pattern.

The correlation between the mitochondrial factor, cell cytokines and the six scale scores in two groups. As is showed in Table V: in the kidney deficiency group: a) the concentration of IL-6 had a negative correlation with the score of MMSE, Barthel index; a positive correlation existed between the concentration of IL-6 and TCM syndrome score, BBS and CDR. b) The concentration of IL-18 negatively related to the score of MMSE, Barthel index and HIS; a positive correlation with CDR. c) The concentration of TNF- $\alpha$ had a negative correlation with the score of MMSE, Barthel index; a positive correlation between the concentration of TNF- $\alpha$ and TCM syndrome score. d) The concentration of SOD negatively related to the TCM syndrome score. e) The concentration of MDA had a positive relation with TCM syndrome score, BBS and CDR. In phlegm turbidity blocking orifice pattern group: a) the concentration of IL-6 had a positive correlation with TCM syndrome score. b) The concentration of IL-18 negatively related to TCM syndrome score, Barthel index, HIS; a positive correlation with BBS. c) A negative correlation between the concentration of TNF- $\alpha$ and Barthel index, the TCM syndrome score positively related to HIS. d) The concentration of SOD negatively related to HIS and the TCM syndrome score, and had a positive correlation with MMSE, BBS; e) the concentration of MDA had a positive relation with the TCM syndrome score.

Multiple linear regression analysis between TCM syndrome score and related factors (IL-6, MDA, SOD, TNF- $\alpha$ ). In order to further analyze which related factors make the greatest effect on TCM syndrome score, multiple linear regression analysis was used to analyze the relationship between TCM syndrome score (Y) and MDA, IL-6, SOD and TNF- $\alpha$.

Multiple linear regression analysis on kidney essence deficiency group. Multiple linear regression analysis between TCM syndrome score and related factors (IL-6, MDA, SOD, TNF- $\alpha$ ). The related factors (IL-6, MDA, SOD, TNF- $\alpha$ ) in kidney essence deficiency group were assigned to $\mathrm{X} 1$, $\mathrm{X} 2, \mathrm{X} 3, \mathrm{X} 4$, and then carried out the multiple linear regression analysis with TCM syndrome score (Y); the results are shown in Table VI. From the analysis of variance (ANOVA), $\mathrm{F}=11.784 \mathrm{P}<0.05$, there was a statistical significance in the regression equation. Finally, IL-6 and SOD were two variables in the regression equation, thus, the regression equation: $\mathrm{Y}=33.085+0.309 \times$ IL-6-0.138 $\times$ SOD.

Multiple linear regression analysis between MMSE and related factors (IL-6, MDA, SOD, TNF- $\alpha$ ). The related factors (IL-6, MDA, SOD, TNF- $\alpha$ ) in kidney essence insufficiency pattern were assigned to $\mathrm{X} 1, \mathrm{X} 2, \mathrm{X} 3, \mathrm{X} 4$, and then the multiple linear regression analysis with MMSE (Y) carried out.The results are shown in Table VII. From the analysis of variance (ANOVA), $\mathrm{F}=16.191 \mathrm{P}<0.05$, there was a statistical significance 
Table VII. Multiple linear regression analysis on MMSE in the kidney essence deficiency group.

\begin{tabular}{|c|c|c|c|c|c|}
\hline \multirow[b]{2}{*}{ Model } & \multicolumn{2}{|c|}{$\begin{array}{l}\text { Non-standard } \\
\text { coefficient }\end{array}$} & \multirow[b]{2}{*}{$\begin{array}{c}\text { Standard } \\
\text { coefficient }\end{array}$} & \multirow[b]{2}{*}{ t-value } & \multirow[b]{2}{*}{ P-value } \\
\hline & B & $\begin{array}{c}\text { Standard } \\
\text { error }\end{array}$ & & & \\
\hline Quantity & 17.949 & 1.225 & & 14.655 & $<0.05$ \\
\hline TNF- $\alpha$ & -0.357 & 0.089 & -0.523 & -4.024 & $<0.05$ \\
\hline
\end{tabular}

Dependent variable: MMSE in kidney essence deficiency group.

Table VIII. Multiple linear regression analysis on BBS the in kidney essence deficiency group.

\begin{tabular}{|c|c|c|c|c|c|}
\hline \multirow[b]{2}{*}{ Model } & \multicolumn{2}{|c|}{$\begin{array}{l}\text { Non-standard } \\
\text { coefficient }\end{array}$} & \multirow[b]{2}{*}{$\begin{array}{l}\text { Standard } \\
\text { coefficient }\end{array}$} & \multirow[b]{2}{*}{ t-value } & \multirow[b]{2}{*}{ P-value } \\
\hline & B & $\begin{array}{l}\text { Standard } \\
\text { error }\end{array}$ & & & \\
\hline Quantity & 6.686 & 2.952 & & 2.264 & 0.029 \\
\hline MDA & 1.923 & 0.830 & 0.333 & 2.318 & 0.025 \\
\hline
\end{tabular}

Dependent variable: BBS in kidney essence deficiency group.

in the regression equation. Finally, TNF- $\alpha$ was the only variable in the regression equation; thus, the regression equation: $\mathrm{Y}=17.949-0.357 \times \mathrm{TNF}-\alpha$.

Multiple linear regression analysis between $B B S$ and related factors (IL-6, MDA, SOD, TNF- $\alpha$ ). The related factors (IL-6, MDA, SOD, TNF- $\alpha$ ) in kidney essence insufficiency pattern were assigned to $\mathrm{X} 1, \mathrm{X} 2, \mathrm{X} 3, \mathrm{X} 4$, and then the multiple linear regression analysis with TCM syndrome score (Y) was carried out; the results are shown in Table VIII. From the analysis of variance (ANOVA), $\mathrm{F}=5.373 \mathrm{P}=0.025$, there was a statistical significance in the regression equation. Finally, MDA was the only variable in the regression equation; thus, the regression equation: $\mathrm{Y}=6.686+1.923 \times \mathrm{MDA}$.

Multiple linear regression analysis between Bathel and related factors (IL-6, MDA, SOD, TNF- $\alpha$ ). The related factors (IL-6, MDA, SOD, TNF- $\alpha$ ) in kidney essence deficiency pattern were assigned to $\mathrm{X} 1, \mathrm{X} 2, \mathrm{X} 3, \mathrm{X} 4$, and then the multiple linear regression analysis with TCM syndrome integral (Y) was carried out; the results are shown in Table IX. From the analysis of variance (ANOVA), $\mathrm{F}=5.228 \mathrm{P}=0.009$, there was a statistical significance in the regression equation. Finally, MDA and IL-6 were two variables in the regression equation; thus, the regression equation: $\mathrm{Y}=97.494-0.836 \mathrm{x}$ IL-6 - 7.285 x MDA.

Multiple linear regression analysis between TCM syndrome score and related factors (IL-6, MDA, SOD, TNF- $\alpha$ ). The related factors (IL-6, MDA, SOD, TNF- $\alpha$ ) in phlegm turbidity
Table IX. Multiple linear regression analysis on Bathel in kidney essence deficiency group.

\begin{tabular}{|c|c|c|c|c|c|}
\hline \multirow[b]{2}{*}{ Model } & \multicolumn{2}{|c|}{$\begin{array}{c}\text { Non-standard } \\
\text { coefficient }\end{array}$} & \multirow[b]{2}{*}{$\begin{array}{l}\text { Standard } \\
\text { coefficient }\end{array}$} & \multirow[b]{2}{*}{ t-value } & \multirow[b]{2}{*}{ P-value } \\
\hline & B & $\begin{array}{l}\text { Standard } \\
\text { error }\end{array}$ & & & \\
\hline Quantity & 97.494 & 14.459 & & 6.743 & $<0.05$ \\
\hline IL-6 & -0.836 & 0.326 & -0.355 & -2.567 & 0.014 \\
\hline MDA & -7.285 & 3.932 & -0.256 & -1.853 & 0.071 \\
\hline
\end{tabular}

Dependent variable: Bathel in kidney essence deficiency group.

Table X. Multiple linear regression analysis on TCM syndrome score in the phlegm turbidity blocking orifice group.

\begin{tabular}{|c|c|c|c|c|c|}
\hline \multirow[b]{2}{*}{ Model } & \multicolumn{2}{|c|}{$\begin{array}{l}\text { Non-standard } \\
\text { coefficient }\end{array}$} & \multirow[b]{2}{*}{$\begin{array}{l}\text { Standard } \\
\text { coefficient }\end{array}$} & \multirow[b]{2}{*}{ t-value } & \multirow[b]{2}{*}{ P-value } \\
\hline & B & $\begin{array}{l}\text { Standard } \\
\text { error }\end{array}$ & & & \\
\hline Quantity & 34.584 & 3.989 & & 8.419 & $<0.05$ \\
\hline TNF- $\alpha$ & 0.212 & 0.092 & 0.249 & 2.316 & 0.024 \\
\hline SOD & -0.137 & 0.032 & -0.448 & -4.310 & $<0.05$ \\
\hline IL-6 & 0.350 & 0.152 & 0.232 & 2.310 & 0.024 \\
\hline
\end{tabular}

Dependent variable: Bathel in phlegm turbidity blocking orifice group.

Table XI. Multiple linear regression analysis on MMSE in the phlegm turbidity blocking orifice group.

\begin{tabular}{lccccc}
\hline \multicolumn{5}{c}{} & \multicolumn{2}{c}{$\begin{array}{c}\text { Non-standard } \\
\text { coefficient }\end{array}$} & & & \\
\cline { 2 - 4 } Model & B & $\begin{array}{c}\text { Standard } \\
\text { error }\end{array}$ & $\begin{array}{c}\text { Standard } \\
\text { coefficient }\end{array}$ & t-value & P-value \\
\hline Quantity & 9.969 & 2.717 & & 3.669 & 0.001 \\
SOD & 0.058 & 0.025 & 0.285 & 2.320 & 0.024
\end{tabular}

Dependent variable: MMSE in phlegm turbidity blocking orifice group.

blocking orifice pattern were assigned to X1, X2, X3, X4, and then the multiple linear regression analysis with TCM syndrome score (Y) was carried out (Table X). From the analysis of variance (ANOVA), $F=16.017$, there was a statistical significance in the regression equation. Finally, IL-6, SOD, TNF- $\alpha$ were variables in the regression equation; thus, the regression equation: $\mathrm{Y}=33.584+0.350 \times$ IL-6 + 0.212 x TNF- $\alpha-0.137 \times$ SOD Table XI. The related factors (IL-6, MDA, SOD, TNF- $\alpha$ ) of 
Table XII. Multiple linear regression analysis on HIS in the phlegm turbidity blocking orifice group.

\begin{tabular}{lcccccc}
\hline & \multicolumn{2}{c}{$\begin{array}{c}\text { Non-standard } \\
\text { coefficient }\end{array}$} & & & \\
\cline { 2 - 4 } Model & B & $\begin{array}{c}\text { Standard } \\
\text { error }\end{array}$ & $\begin{array}{c}\text { Standard } \\
\text { coefficient }\end{array}$ & t-value & P-value \\
\hline Quantity & 17.280 & 0.907 & & 19.047 & $<0.05$ \\
SOD & -0.036 & 0.010 & -0.423 & -3.643 & 0.001 \\
\hline
\end{tabular}

Dependent variable: HIS in phlegm turbidity blocking orifice group.

MMSE in phlegm turbidity blocking orifice group were assigned to $\mathrm{X} 1, \mathrm{X} 2, \mathrm{X} 3, \mathrm{X} 4$, and then carried out the multiple linear regression analysis with TCM syndrome score (Y) (Table XII). From the analysis of variance (ANOVA), $\mathrm{F}=5.382$ $\mathrm{P}=0.024$, there was a statistical significance in the regression equation. Finally, SOD was the only variable in the regression equation; thus, the regression equation: $\mathrm{Y}=9.969+0.058 \times$ SOD. The related factors (IL-6, MDA, SOD, TNF- $\alpha$ ) of HIS in phlegm turbidity blocking orifice pattern were assigned to $\mathrm{X} 1, \mathrm{X} 2, \mathrm{X} 3, \mathrm{X} 4$, and then carried out the multiple linear regression analysis with TCM syndrome score (Y). From the analysis of variance (ANOVA), $F=13.274$ $\mathrm{P}=0.001$, there was a statistical significance in the regression equation. Finally, SOD was the only variable in the regression equation; thus, the regression equation: $\mathrm{Y}=17.843-0.036 \times \mathrm{SOD}$.

\section{Discussion}

The decline of behavior and ability of daily living in the kidney essence deficiency group is more severe than that in phlegm turbidity blocking orifice group. Kidney is the source of acquired constitution, governing bone and producing marrow. Kidney $q i$ contact with the brain that is house of bright essence which charge the human high nervous activity. According to TCM, the human's kidney qi decline at septuple eight, the shortage of Yang Ming pulse, exhausted heavenly tenth, brain constraint, which could result in the decline of behavior and ability of daily living. In addition, the causes of liver $q i$ stagnation, insufficiency of kidney essence, dual deficiency of yin and yang of kidney, dual deficiency qi and blood of spleen - heart, impaired lung depuration that could lead to the operational obstacle of $q i$, blood and body fluid in body. In consequence, the phlegm is produced in the resulting of $q i$ constraint, spleen failing to transport and transform. Phlegm disturb the body fluid route and it could be mixed with other tangible pathogenic products, producing new etiology and pathogenesis such as the fusion of phlegm stasis in brain, the mental confusion caused by wind phlegm, the damaged brain as result of phlegm fire, collectively leading to brain malnutrition are observed. When the brain lose its normal function, decreasing memory and viability, disfunction in comprehension and execution. The results showed that the scores of CDR and levels of memory, judgment, social affairs, family+hobby had significantly differences in the groups, the means of kidney essence deficiency group were higher than those of phlegm turbidity blocking orifice pattern, which showed the degree of CDR in sthenic syndrome group was more severe than that of in asthenic syndrome group. The mean of personal care in BBS in kidney essence deficiency group was higher than that of phlegm turbidity blocking orifice group. There was a negative correlation between TCM syndrome score and MMSE, Barthel Index in kidney essence deficiency group. In asthenic syndrome group, the higher scores of MMSE, and PSMS were, the lower the scores in TCM symptoms. The TCM symptom scale scores had a negative correlation with BBS in phlegm turbidity blocking orifice group.

The results above showed that there was no difference on cognition between sthenia syndrome and asthenia syndrome of VD. However, the decline on behavior and ability of daily living of sthenia syndrome group were more obvious than that of in sthenia syndrome group. Numerous experiments in vivo and in vitro implicated that inflammation is involved in the pathological mechanism of VD. Some studies found inflammatory cellular factors mainly include the IL- 6 , IL-18, TNF- $\alpha$ and IL-1 $\beta$ that take part in inflammation of cognitive disorder after stroke.

TNF- $\alpha$ and IL- 6 are pro-inflammatory cytokines activated in response to infection or injury by activating macrophages, lymphocytes and other immune and structural cells including astrocytes and neurons $(8,9)$. TNF- $\alpha$ and IL- 6 can be a starter of the inflammatory cascade, which could cause the secondary injury in the brain. Some research show that IL-6 may be the biological marker that affect intelligence in cerebrovascular diseases although others research did not find high IL-6 might be a specific marker (10).

To verify the results of these studies, we found the serum TNF- $\alpha$ and IL-6 levels were increased in these two groups, indicating a pro-inflammatory status in VD patients. This may suggest the mechanism of this change, which inflammation may be associated with the physiopathology of VD. Moreover, IL-6 levels were lower in patients with phlegm turbidity blocking orifice group than that of with kidney essence deficiency group which may be novel marker in TCM syndrome.

IL-18 can stimulate cell proliferation and inhabit the death of the NK by regulating a variety of signal transduction pathways, as a result of inducing the production of interferon- $\gamma$ (IFN- $\gamma$ ), which could damage the brain for releasing a large number of inflammatory factors. Mitochondrion damage caused by oxidative stress was also involved in the pathological mechanism of VD. It is worth mentioning that this research also proved that the mitochondrial damage caused by inflammatory reactions and over-oxidation might be involved in the process of VD in the two groups. The means of IL-18 and SOD in phlegm turbidity blocking orifice group were higher than that of kidney essence deficiency group, which was in accord with the results of previous studies. In a previous study, the level of IL-18 in patient's blood was significantly higher than that of the control group, reminding that IL-18 may be involved in the inflammation of VD. At present, some researchers put the certain inflammatory factors as the targets of research for clinical drug research. Previous evidence showed that the VD is treated by Wenpi Tongluo decoction and Nimodipine, the study found the level of IL- 6 and TNF- $\alpha$ would be decreased 
by the combined treatments of TCM and western medicine, and there is a significantly increase of the scores of MMSE after treatments. This result was in accord with higher serum ILs were associated with poor cognitive status, including poor performance in memory and processing speed, as well as cognitive decline $(10,11)$.

With the development of the integration of traditional and western medicine, the realization is of VD developing from macroscopic to microcosmic, from basic theory of TCM to the combination of experimental pathology research of western medicine and the theory system of TCM. Yongyan Wang proposed the theory of 'toxin damaging brain collaterals' to the stroke and VD. 'Toxin' is invasive, creeping, easy moving, if the toxin is lingering in our brain, it could lead to cognitive disorder.

As shown in the study, the higher scores of MMSE in kidney essence deficiency group were closely correlated with the lower levels of TNF- $\alpha$, IL-6, and the higher scores of Barthel index were related to the lower values of the TNF- $\alpha$, IL- 6 and MDA; inflammatory factors and free radicals aggravate the degree of dementia. The higher the scores of TCM symptom scale were, the higher values of TNF- $\alpha$, IL-6 were, and the lower the values of SOD that play an antioxidant effects, the weaker abilities of antioxidant in patients were in kidney essence deficiency group. The higher the scores of BBS and CDR were related to the higher values of IL-6, MDA in kidney essence deficiency group. The higher scores of TCM syndrome integral were related to the higher values of the TNF- $\alpha$, IL-6 and MDA, and the lower value of SOD that play an antioxidant effects, the weaker the abilities of antioxidant in patients were in phlegm turbidity blocking orifice pattern group. There was a positive relation between TNF- $\alpha$ and HIS, which played an important role in ischemic access, and SOD reduce the degree of ischemia in phlegm turbidity blocking orifice pattern group. From the results of this study, the mean of IL-18 in phlegm turbidity blocking orifice group was higher than that of kidney essence deficiency group.

As an important endogenous antioxidantive enzyme in the body, SOD is one of the main enzymes with the inhibitation of free radical reaction, which could transfer superoxide anion to hydrogen peroxide. The results of this study showed that the phlegm turbidity blocking orifice pattern group, the phlegm could be transformed into the toxin that could lead to the disfunction of the brain, causing continuous inflmmation in the body, as a result of stimulating the production of SOD in the body, the higher level of IL-18 promotes the progress of dementia. This research showed there was a positive correlation between TCM syndrome score and the level of IL-6, IL-8, TNF- $\alpha$, and MDA, a negative relation with SOD in two groups.

This result was consistent with the theory of 'toxin damaging brain collaterals', 'toxin' include a lot of hazardous substances produced in the progression of the pathology from the disorder of energy metabolism which was caused by the ischemia and hypoxia in the brain, In other words, 'toxin' was not only the production of inflammation, but was an intermediate cause of oxidative stress. From the perspective of Traditional Chinese Medicine, this 'toxin' comes from the body or out of the body, and the 'toxin' of body can be derived from phlegm evil except from wind, fire and blood stasis. Study showed that kidney deficiency and phlegm stasis are the basis pathogenesis of VD (12). Moreover, the key factor is accumulation of phlegm and blood stasis, phlegm and toxin producing. Because the patients belong to kidney essence insufficiency pattern of VD, deficiency in kidney already existed, but phlegm and blood stasis were not obvious. For the patients who belong to sthenia syndrome in phlegm group, because of the existence of phlegm, the phlegm could be transformed into the toxin that could lead to the disfunction of the brain day by day, finally causing inflammation in the body. Thus, the level of IL-18 in phlegm group was higher than that of asthenia syndrome.

There had an obvious relevance between the index of inflammatory factors, related mitochondrion factors and TCM syndrome integral. Therefore, we could reduce the effects of 'toxin' on the brain and slow down or prevent the occurrence and delay development of VD. Previously researchers (7) used FuZhi capsule that could tonify the kidney essence and eliminate phlegm based on the theory of 'toxic impaired brain retinervus', research showed that FuZhi capsule can reduce the level of TNF- $\alpha$, IL- 6 and MDA, and also increase and improve the cognitive ability.

The kidney essence insufficiency pattern and phlegm turbidity blocking orifice pattern are common syndromes for VD, which are representative of asthenia syndrome and sthenia syndrome. The results of this research were consistent with previous studies, that IL-6, IL-18, MDA and TNF- $\alpha$ were involved in the inflammation and mitochodrial damage may be involved in the process of vascular dementia. The higher the inflammation was, the more serious mitochondrion damage was, the higher the scores of the TCM syndrome score were, the more serious the 'toxin' was in the two groups. Besides, the level of IL-18 in the phlegm turbidity blocking orifice pattern was higher than that of the kidney essence insufficiency pattern, and make more important contributions to TCM symptom score; the level of IL- 6 in the kidney essence insufficiency pattern was higher than that of the phlegm turbidity blocking orifice pattern, and the lower activity of SOD made greater contributions to TCM symptom score. Therefore, all the above results would be of great significance for clinic and research. From the view of the clinic, due to the dialectic of TCM for VD had certain subjectivity, we could treat the disease based on the finding of decreasing the level of IL-18 in phlegm turbidity blocking orifice pattern and decreasing the level of IL-6, increasing the activity of SOD in kidney deficiency syndrome. Therefore, the patients who belong to kidney essence insufficiency pattern could take IL-6 as the main target for anti-inflammatory treatmen, and the patients in phlegm turbidity blocking orifice pattern group should put IL-18 as the main target for anti-inflammation, and improve the level of SOD which promote the ability of anti-oxidation in the body.

\section{References}

1. Zhou M, Xia ZY, Lei SQ, Leng Y and Xue R: Role of mitophagy regulated by Parkin/DJ-1 in remote ischemic postconditioninginduced mitigation of focal cerebral ischemia-reperfusion. Eur Rev Med Pharmacol Sci 19: 4866-4871, 2015.

2. Bennett S, Grant MM and Aldred S: Oxidative stress in vascular dementia and Alzheimer's disease: A common pathology. J Alzheimers Dis 17: 245-257, 2009 
3. Jeon GS, Park SK, Park SW, Kim DW, Chung CK and Cho SS Glial expression of interleukin-18 and its receptor after excitotoxic damage in the mouse hippocampus. Neurochem Res 33 $179-184,2008$

4. Chi CL, Zhang SA, Liu Z, Chang MX, Wang H and Huang Y: Research on the role of GLP-2 in the central nervous system EPK signal transduction pathway of mice with vascular dementia. Eur Rev Med Pharmacol Sci 21: 131-137, 2017.

5. Lang UE and Borgwardt S: Molecular mechanisms of depression: Perspectives on new treatment strategies. Cell Physiol Biochem 31: 761-777, 2013.

6. Yagami T:Cerebral arachidonatecascadeindementia:Alzheimer's disease and vascular dementia. Curr Neuropharmacol 4: 87-100, 2006.

7. Yang Y, Wang L, Wu Y, Su D, Wang N, Wang J, Shi C, Lv L and Zhang S: Tanshinol suppresses inflammatory factors in a rat model of vascular dementia and protects LPS-treated neurons via the MST1-FOXO3 signaling pathway. Brain Res 1646: 304-314, 2016.

8. Malaguarnera L, Motta M, Di Rosa M, Anzaldi M and Malaguarnera M: Interleukin-18 and transforming growth factor-beta 1 plasma levels in Alzheimer's disease and vascular dementia. Neuropathology 26: 307-312, 2006.
9. Zhang XL, Zheng SL, Dong FR and Wang ZM: Nimodipine improves regional cerebral blood flow and suppresses inflammatory factors in the hippocampus of rats with vascular dementia. J Int Med Res 40: 1036-1045, 2012.

10. Huberman M, Sredni B, Stern L, Kott E and Shalit F: IL-2 and IL-6 secretion in dementia: Correlation with type and severity of disease. J Neurol Sci 130: 161-164, 1995.

11. Zhou X, Cui G, Tseng HH, Lee SM, Leung GP, Chan SW, Kwan YW and Hoi MP: Vascular contributions to cognitive impairment and treatments with traditional Chinese medicine. Evid Based Complement Alternat Med 2016: 9627258, 2016.

12. Yu TM, Chuang YW, Sun KT, Yu MC, Kung SC, Lee BK, Huang ST, Chen $\mathrm{CH}$, Lin $\mathrm{CL}$ and $\mathrm{Kao} \mathrm{CH}$ : Polycystic kidney disease is significantly associated with dementia risk. Neurology 89: 1457-1463, 2017. Attribution-NonCommercial-NoDerivatives 4.0 International (CC BY-NC-ND 4.0) License. 